\title{
A New Interface Defect Spectroscopy Method
}

\author{
J.T. Ryan ${ }^{1}$, L.C. Yu ${ }^{1,2}$, J.H. Han ${ }^{1}$, J.J Kopanski ${ }^{1}$, K.P. Cheung ${ }^{1, *}$, F. Zhang ${ }^{3}$, C. Wang ${ }^{4,5}$, \\ J.P. Campbell ${ }^{1}$, J.S. Suehle ${ }^{1}$, V. Tilak ${ }^{2}$, and J. Fronheiser ${ }^{2}$ \\ ${ }^{1}$ National Institute of Standards and Technology, Gaithersburg, MD \\ ${ }^{2}$ GE Global Research, Niskayuna, NY \\ ${ }^{3}$ Michigan State University, East Lansing, MI \\ ${ }^{4}$ Purdue University, West Lafayette, ID \\ ${ }^{5}$ Fudan University, Shanghai, China \\ *301-975-3093, kin.cheung@nist.gov
}

\begin{abstract}
A new interface defect spectroscopy method based on variable height charge pumping capable of observing the amphoteric nature of $\mathrm{Si} / \mathrm{SiO}_{2}$ interface states in production quality sub-micron devices is demonstrated. It can help to resolve the long standing debate about the true nature of $\mathrm{Si} / \mathrm{SiO}_{2}$ interface states. Additionally, we show that this is a powerful technique for studying other important material systems.
\end{abstract}

\section{Keywords; interface states, $P_{b}$ centers, charge pumping}

\section{INTRODUCTION}

There is a long standing debate on whether $\mathrm{P}_{\mathrm{b}}$ centers account for all electrically observed $\mathrm{Si} / \mathrm{SiO}_{2}$ interface states. The inability to establish the amphoteric nature of $\mathrm{Si} / \mathrm{SiO}_{2}$ interface states in high quality samples is a key obstacle for resolving this issue. In this work, we introduce a new interface defect spectroscopy method based on variable height charge pumping and report the amphoteric signature of $\mathrm{Si} / \mathrm{SiO}_{2}$ interface states in production quality samples for the first time. The new technique is demonstrated on sub-micron size devices, highlighting the exceptional level of sensitivity.

Our new technique is a modified charge-pumping (CP) measurement. When applied to production quality pure $\mathrm{SiO}_{2}$ MOSFETs with negligible bulk trapping centers, we can be confident that the CP signal is due only to interface states. Fig. 1 shows the key result demonstrating the new technique. The characteristic double peaks (which maintain their shape and increase in magnitude following moderate gate stress) at the expected energy locations clearly show that the interface states are of the $\mathrm{P}_{\mathrm{b}}$ center family. Please note the absolute number of defects (left vertical axis) and the defect density (right vertical axis). This is the first ever reported double peak signature on a high quality sub-micron device.

All previously reported successful electrical observations of the amphoteric nature of $\mathrm{Si} / \mathrm{SiO}_{2} \mathrm{P}_{\mathrm{b}}$ centers relied on devices with very poor quality interfaces $\left(\mathrm{D}_{\mathrm{it}}>10^{11} \mathrm{~cm}^{-2}\right)$ and/or very large device areas [1-14]. In some cases, the amphoteric nature was only observable after very harsh irradiation $[2,7,8,12$, 14]. In addition to a high density of interface states, these samples also exhibit high densities of non-interface bulk trapping centers. As most electrical measurements are not capable of truly excluding non-interface defects, quantitative disagreements are common when compared to $\mathrm{P}_{\mathrm{b}}$ center data obtained from electron spin resonance. Such disagreements fueled the controversy $[15,16]$.
Using CP to perform defect spectroscopy is not new [1-3, 11]. Previous attempts were based on trapped charge emission which had difficulty probing mid-gap and (in some cases) require complicated gate voltage pulse trains that limit access near the band edges (slower pulse rise time $\left(\mathrm{t}_{\mathrm{r}}\right)$ and fall time $\left.\left(t_{f}\right)\right)[1-3,11]$. Our approach utilizes a simple square wave, relies on charge capture rather than emission, is able to probe defects through mid-gap, and allows the use of faster $t_{r}$ and $t_{f}$ to probe closer to the band edges. Additionally, choosing a frequency low enough to ensure complete trap filling is the key new aspect in our approach.

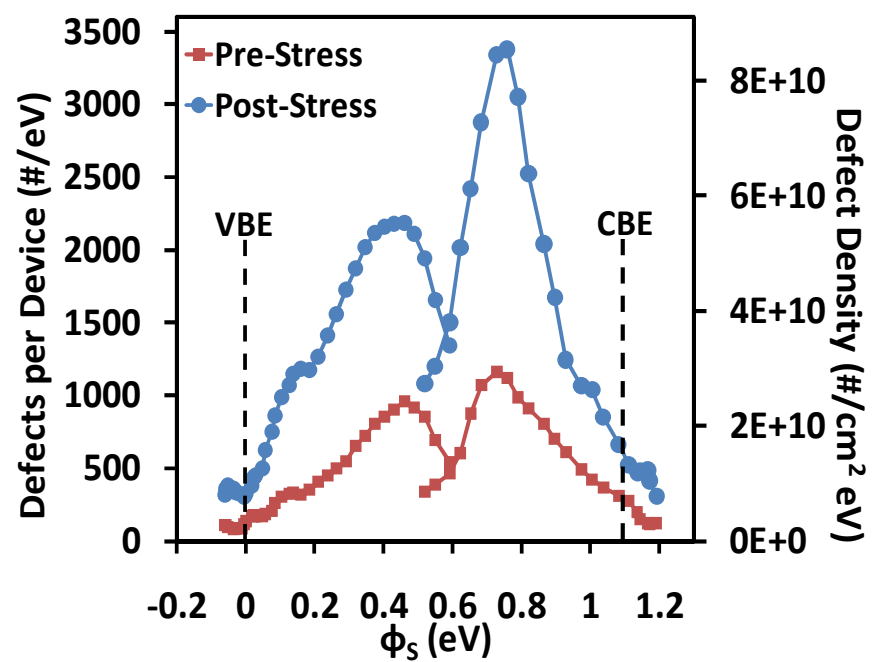

Fig. 1: Amphoteric nature of $\mathrm{Si} / \mathrm{SiO}_{2}$ interface states in a production quality sub-micron device. This is the first ever reported double peak signature on a high quality submicron device.

\section{EXPERIMENTAL}

The devices used are production quality $16.45 \times 0.24 \mu \mathrm{m}^{2}$ nMOSFETs with $5.5 \mathrm{~nm}$ pure $\mathrm{SiO}_{2}$ gate dielectrics $\left(\mathrm{V}_{\text {th }} \approx 0.65\right.$ V). CP was performed by applying a simple square wave (provided by a commercially available pulse generator) to the gate while shorting the source and drain to ground. The substrate (charge pumping) current $\left(\mathrm{I}_{\mathrm{CP}}\right)$ was measured with an ultra-low noise current preamplifier and recorded with a digital storage oscilloscope for several seconds. The CP current was then averaged offline to further increase the signal to noise ratio. This approach allowed us to accurately measure very small currents with excellent sensitivity. Our CP method is 
very similar to the variable pulse height method [17]. The upper half of the band gap is measured by setting the pulse low level $\left(\mathrm{V}_{\mathrm{GL}}\right)$ at strong accumulation $\left(\mathrm{V}_{\mathrm{GL}}=-2 \mathrm{~V}\right.$ in these samples) while sequentially stepping the pulse high level $\left(\mathrm{V}_{\mathrm{GH}}\right)$ from strong inversion to depletion. The lower half of the band gap is measured by setting $\mathrm{V}_{\mathrm{GH}}$ at strong inversion $\left(\mathrm{V}_{\mathrm{GH}}=1 \mathrm{~V}\right.$ in these samples) while sequentially stepping $\mathrm{V}_{\mathrm{GL}}$ from strong accumulation to depletion. By varying the pulse height in this fashion, we can obtain CP current as a function of the probed energy window in the band-gap. Fig. 2 schematically illustrates the pulse conditions. To reduce emission loss and push the measurement window closer to the band edges, $t_{r}$ and $\mathrm{t}_{\mathrm{f}}$ are both held at $2.3 \mathrm{~ns}$ for all measurements (unless otherwise noted). Based on instrumentation limits, slower $t_{r}$ and $t_{f}$ times can be used, but this will result in greater emission loss and will reduce how close to the band edges one can probe. Alternatively, faster $t_{r}$ and $t_{f}$ can be used to push the measurement window closer to the band edges, but care must be taken to avoid CP geometric effects based on the device dimensions as well as waveform distortion. Additionally, for more careful analysis of the band edges, the slope of the pulse transition for various pulse heights should be considered.
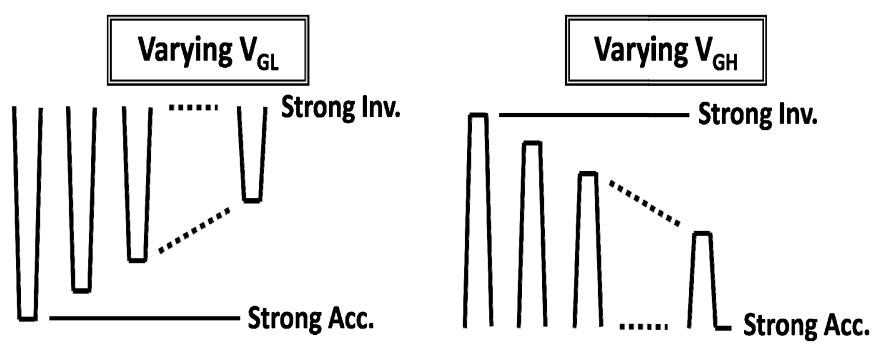

Fig. 2: Schematic illustration of the pulse bias conditions. For the case of fixed strong inversion (left), the pulse low level $\left(\mathrm{V}_{\mathrm{GL}}\right)$ is sequentially stepped from strong accumulation to deep depletion. For the case of fixed strong accumulation (right), the pulse high level $\left(\mathrm{V}_{\mathrm{GH}}\right)$ is sequentially stepped from strong inversion to deep depletion.

For each pulse bias level, the CP current was measured at 1, 2,3 , and $4 \mathrm{kHz}$. Such low frequency is of key importance and separates our approach from the conventional variable pulse height method, as explained later. Since the CP current should be zero at a $\mathrm{CP}$ frequency of $0 \mathrm{~Hz}$, a linear plot of $\mathrm{CP}$ current vs. frequency for each pulse bias level allows for leakage current and amplifier offset to be corrected (by subtracting off the linear fit $\mathrm{y}$-intercept value) and the $\mathrm{CP}$ current at a $2 \mathrm{kHz}$ to be extracted accurately. Fig. 3 illustrates corrected CP current vs. frequency for two different pulse bias levels along with their respective linear fits. In thinner gate dielectrics, correcting for gate leakage current with this approach becomes difficult as the gate leakage current starts to overwhelm the CP current. Since the ratio of gate leakage current versus $\mathrm{CP}$ current can become quite large, this correction looses accuracy and introduces errors in the extracted measurement results. Thus, we estimate that below about $2 \mathrm{~nm}$ of pure $\mathrm{SiO}_{2}$ gate oxide thickness, the gate leakage current becomes large enough such that this method should be used carefully and the results viewed with care.
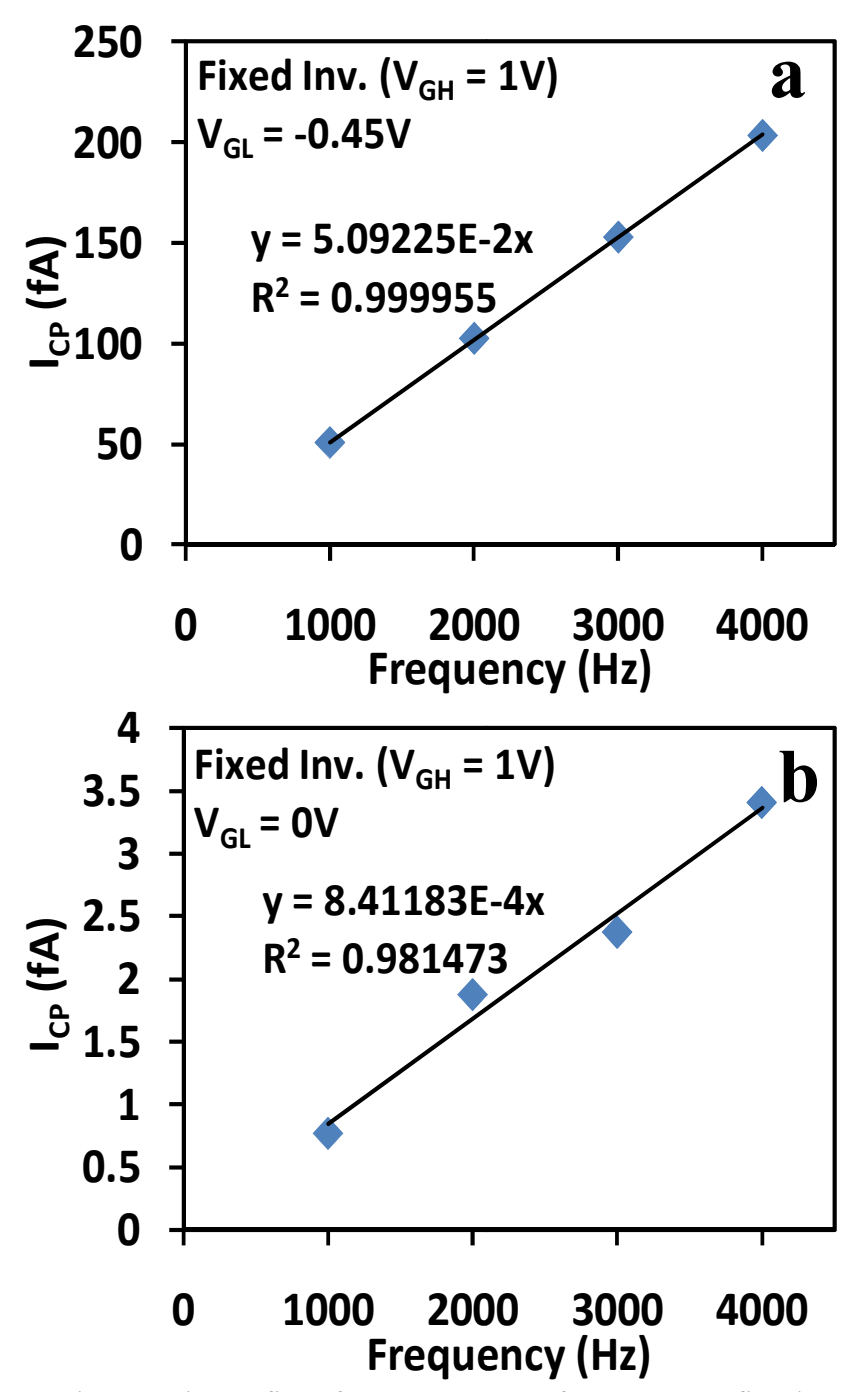

Fig. 3: Linear fits of CP current vs. frequency at fixed inversion for (a) $\mathrm{V}_{\mathrm{GL}}=-0.45 \mathrm{~V}$ and (b) $\mathrm{V}_{\mathrm{GL}}=0 \mathrm{~V}$ after correcting for gate leakage current and amplifier offset.

The linear fit $\left(\mathrm{R}^{2}\right)$ is clearly degraded in (b) indicating the onset of incomplete trap filling, as discussed in the text.

\section{DISCUSSION}

As mentioned above, choosing a CP frequency low enough to ensure complete trap filling is a vital necessity for an accurate measurement. When we vary the pulse height deep into depletion, the carrier density can become very small. Since the time constant associated with trap filling is inversely proportional to the number of carriers available to fill, the trap filling time becomes longer as the pulse height is pushed deep into depletion. This means that traps expected to participate in the $\mathrm{CP}$ process are not filled completely if sufficient time (slow enough frequency) is not given. The result is an apparent loss of measured $\mathrm{CP}$ current which detrimentally alters the measurement result. This is the reason we extract our CP results at $2 \mathrm{kHz}$. 
The specific frequency is experimentally determined by measuring the $\mathrm{CP}$ current as a function of frequency for various pulse bias conditions as shown in Fig. 4 for the case of fixed accumulation (fixed $\mathrm{V}_{\mathrm{GL}}$ ). It clearly demonstrates that as the frequency is increased and/or $\mathrm{V}_{\mathrm{GH}}$ is reduced into depletion, charge per cycle decreases; this is caused by insufficient time to ensure complete filling of the traps within the measurement window and must be avoided.

Charge per cycle is essentially the number of traps participating in the $\mathrm{CP}$ process and is extracted by dividing the measured CP current by the $\mathrm{CP}$ frequency and electronic charge (q). When incomplete trap filling is avoided, we equate "charge per cycle" to "defects per device" since all traps within the probed energy window in the device are contributing to the $\mathrm{CP}$ current. Ensuring the measured result is free from incomplete trap-filling effects separates our new method from conventional variable pulse height $\mathbf{C P}$, which is usually done at higher frequency.

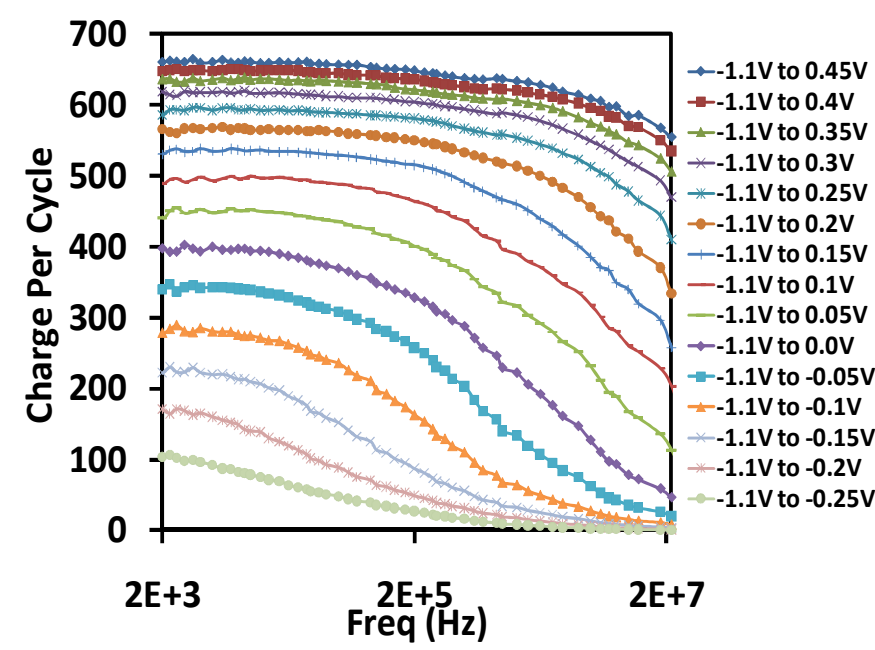

Fig. 4: Charge per cycle vs. frequency at fixed accumulation for a variety of different pulse high levels $\left(\mathrm{V}_{\mathrm{GH}}\right)$. As the CP frequency is increased and/or $\mathrm{V}_{\mathrm{GH}}$ is pushed into depletion, charge loss due to incomplete trap

filling clearly occurs. This effect must be avoided.

Fig. 5 shows the measured defect density $\left(\# / \mathrm{cm}^{2}\right)$ vs. gate voltage $\left(\mathrm{V}_{\mathrm{G}}\right)$ for both fixed inversion and fixed accumulation cases at a frequency of $2 \mathrm{kHz}$. For the fixed accumulation case, $\mathrm{V}_{\mathrm{G}}$ indicates the stepped pulse high level $\left(\mathrm{V}_{\mathrm{GH}}\right)$ and for the fixed inversion case, $V_{\mathrm{G}}$ indicates the stepped pulse low level $\left(\mathrm{V}_{\mathrm{GL}}\right)$. To obtain the defect density, defects per device (explained above) is extracted at $2 \mathrm{kHz}$ and is then divided by the device area. Superimposed in Fig. 5 is the simulated [18] pre-stress capacitance vs. $\mathrm{V}_{\mathrm{G}}(\mathrm{CV})$ curve.

It is clear that the defect density in Fig. 5 refers to the total defects within the probed energy window. As we vary $\mathrm{V}_{\mathrm{GL}}$ (or $\mathrm{V}_{\mathrm{GH}}$ ) we change the probed energy window. By taking the derivative of the measured defect density with respect to $V_{G}$, we can extract the density of states (DOS) of the traps participating in the $\mathrm{CP}$ process. This derivative is shown in Fig. 6 (the pre-stress curves of Fig. 1) after converting $V_{G}$ to silicon surface potential $\left(\phi_{\mathrm{s}}\right)$ using the simulated CV curve (superimposed). The left hand curve (extracted from fixed inversion) peaks at about $0.45 \mathrm{eV}$ above the valence band edge (VBE) and the right hand curve (extracted from fixed accumulation) peaks at about $0.76 \mathrm{eV}$ above the VBE.

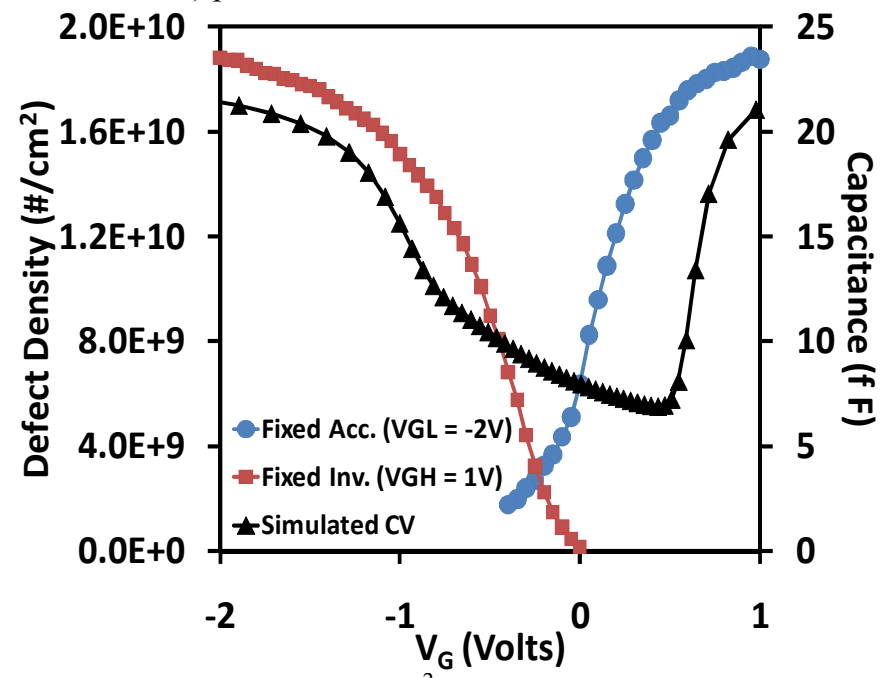

Fig. 5: Defect density $\left(\# / \mathrm{cm}^{2}\right)$ vs. $\mathrm{V}_{\mathrm{G}}$ extracted from the CP current at $2 \mathrm{kHz}$ and a superimposed simulated $\mathrm{CV}$ curve. $\mathrm{V}_{\mathrm{G}}$ indicates pulse high level $\left(\mathrm{V}_{\mathrm{GH}}\right)$ and low level $\left(\mathrm{V}_{\mathrm{GL}}\right)$ for fixed accumulation and fixed inversion cases, respectively.

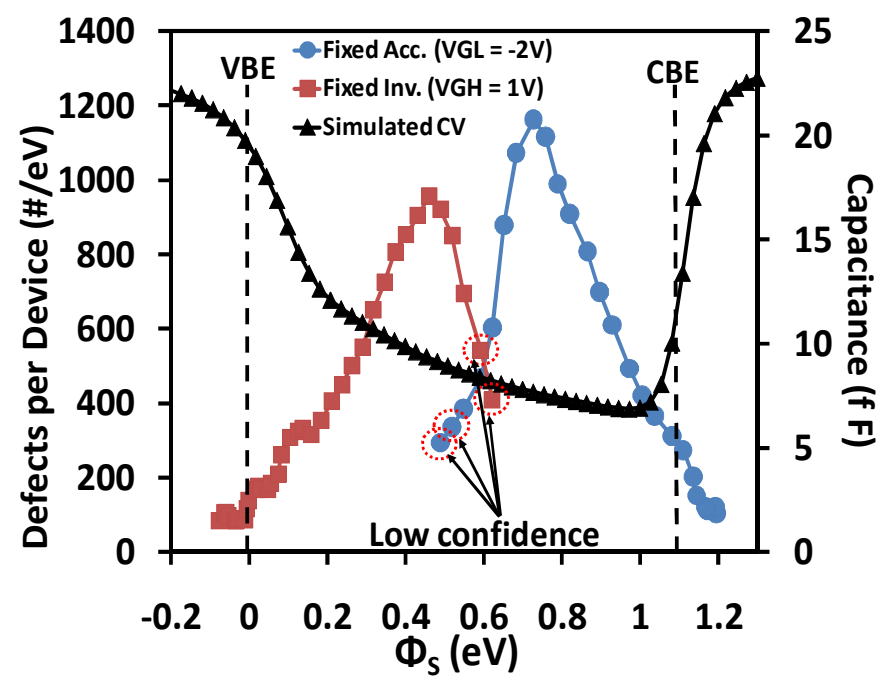

Fig. 6: DOS illustration vs. silicon surface potential $\left(\phi_{\mathrm{S}}\right)$ and a simulated CV curve. The DOS (\#/eV) was computed by taking the derivative of the data from Fig. 5 with respect to $\mathrm{V}_{\mathrm{G}}$ and $\phi_{\mathrm{S}}$ was calculated from the simulated $\mathrm{CV}$ curve.

The indicated data points have low confidence, as explained in the text.

An obvious question is why the two sides of the measured DOS are not in agreement where they overlap? The answer involves the accuracy of the measurement falling off as the pulse height is pushed deep into depletion. As mentioned above, for each pulse bias condition, frequency dependent $\mathrm{CP}$ was performed at frequencies of $1,2,3$, and $4 \mathrm{kHz}$, and the $\mathrm{CP}$ current at $2 \mathrm{kHz}$ was extracted from a linear fit correction. As we push deep into depletion, incomplete trap filling starts to set in (as illustrated in Fig. 4) and the linearity of the frequency dependent $\mathrm{CP}$ data will start to degrade (the linear fit $\mathrm{R}^{2}$ value 
in Fig. $3 b$ is worse than in Fig. $3 a$ which is in part due to this). Fig. 7a compares the linear fit $\mathrm{R}^{2}$ values for fixed inversion with $\mathrm{V}_{\mathrm{GL}}=-0.7 \mathrm{~V}$ to $0 \mathrm{~V}$. At certain $\mathrm{V}_{\mathrm{GL}}$ levels (as we push deeper into depletion), the linear fit $\mathrm{R}^{2}$ value clearly starts to degrade. This is indicative of the on-set of incomplete trap filling (as well as signal to noise degradation). Similarly, the phenomenon is also clearly observed in the fixed $\mathrm{V}_{\mathrm{GL}}$ case of Fig. 7b. This $\mathrm{R}^{2}$ approach provides a convenient, objective and stringent method to monitor incomplete trap filling issues.
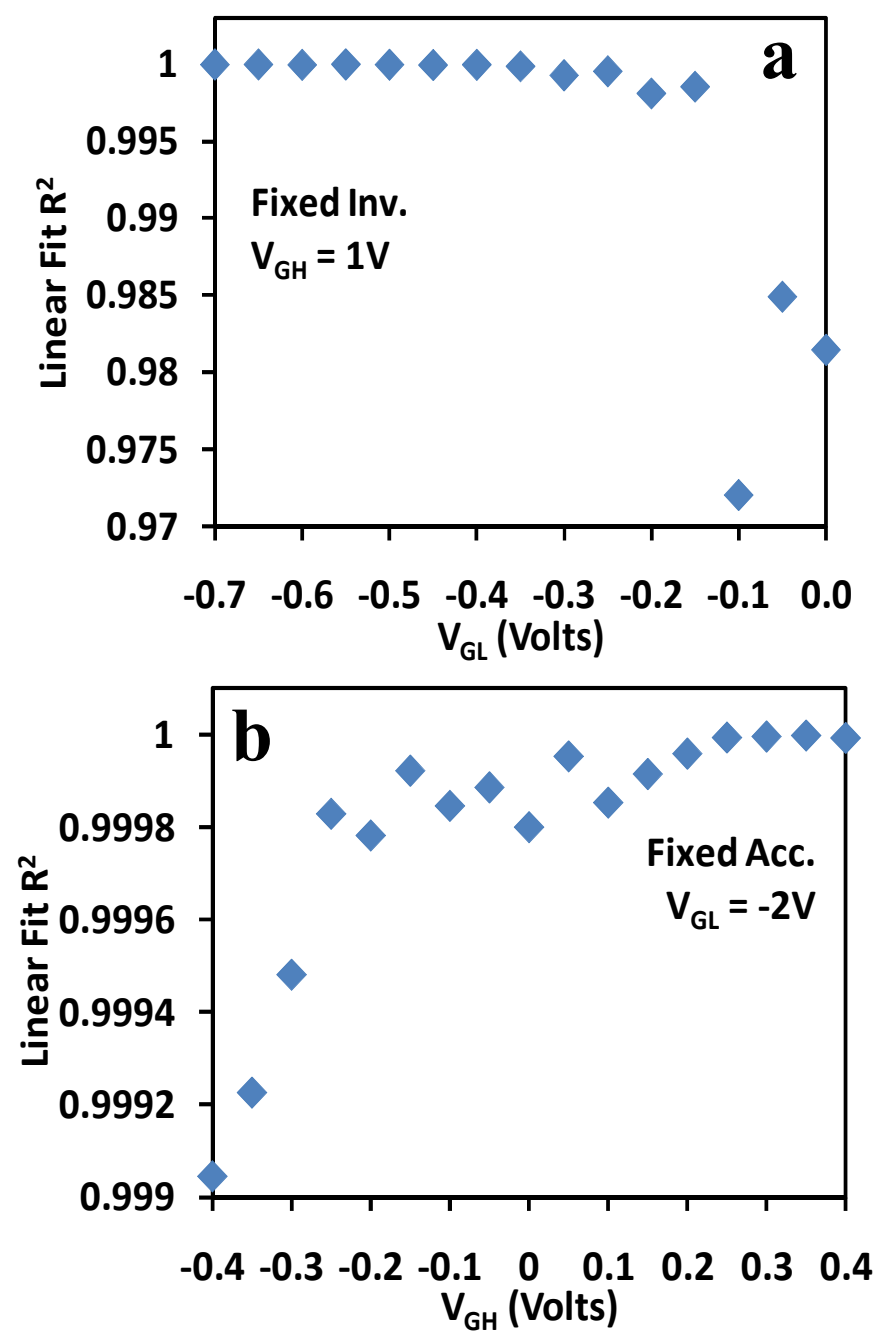

Fig. 7: Liner fit quality $\left(\mathrm{R}^{2}\right)$ vs. pulse height for fixed inversion (a) and fixed accumulation (b). As the pulse height is decreased, the linear fit is degraded; the degraded data has low confidence.

Clearly, the reliability of the last three points in Fig. 7a and the first three data points in Fig. $7 \mathrm{~b}$ should not be trusted. These points are already dropped in Figs. 1 and 6. However, our differentiation routine propagates the influence of these points to the next three points. (We use the Savitzky-Golay method for differentiation, which is formally equivalent to taking a small segment of the curve and fitting it to a second order polynomial, and then differentiating the polynomial [19].) As differentiation tends to amplify small errors, the influence at the first point is significant. The next point is less. From the fourth point on, the influence is no longer there.
Based on this consideration, we circle the data points with a low confidence level in Fig. 6, thereby eliminating the disagreement.

To illustrate the new technique and to show that the peak locations are not measurement artifacts, we apply this technique to a different material system, namely $\mathrm{SiC} / \mathrm{SiO}_{2}$. For this system, one would intuitively expect a different DOS distribution than conventional $\mathrm{Si} / \mathrm{SiO}_{2}$ as $\mathrm{SiC} / \mathrm{SiO}_{2}$ could have silicon dangling bonds as well as carbon dangling bonds each with two DOS peaks. Fig. 8 shows the results of applying our new technique on a SiC nMOSFET $\left(400 \times 2 \mu \mathrm{m}^{2}, \mathrm{t}_{\mathrm{ox}}=50 \mathrm{~nm}\right)$ and four peaks are observed. Measured CV curves are superimposed and the $\mathrm{VBE}$ and $\mathrm{CBE}$ markers are estimates only. In this figure, the DOS was extracted at $10 \mathrm{kHz}$, which was experimentally determined to avoid the interface trap filling time issue discussed above, and $t_{r}$ and $t_{f}$ were both held at $50 \mathrm{~ns}$ to avoid CP geometric effects. This result illustrates the power of this technique when studying different material systems as well as strongly supporting the validity of our $\mathrm{Si} / \mathrm{SiO}_{2}$ DOS peaks.

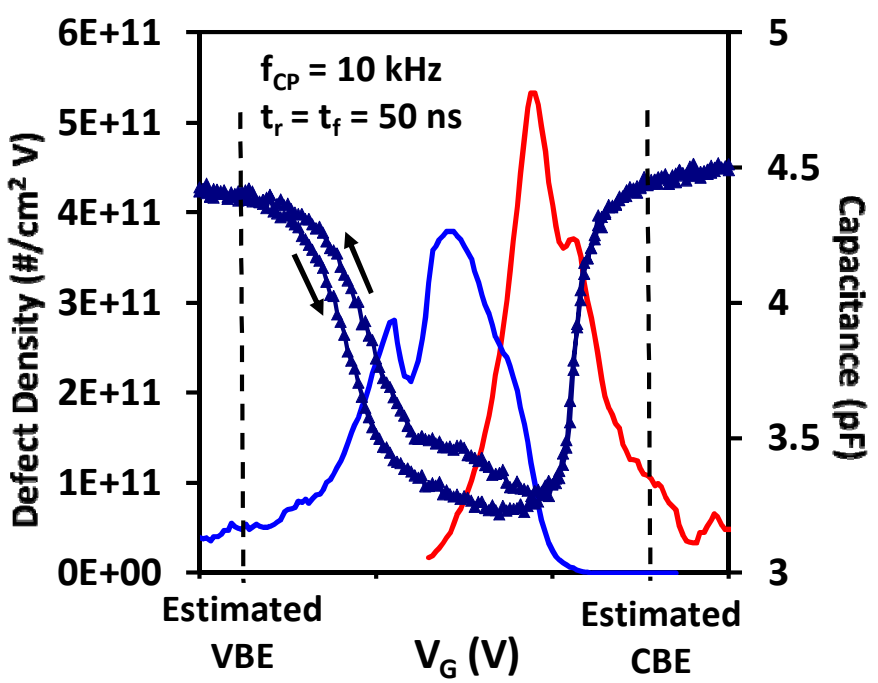

Fig. 8: DOS illustration and a measured $\mathrm{CV}$ curve for a $\mathrm{SiC}$ nMOSFET. Four peaks clearly appear in the DOS which are intuitively due to silicon dangling bonds and carbon dangling bonds.

\section{CONCLUSIONS}

We have shown, for the first time, the amphoteric nature of $\mathrm{Si} / \mathrm{SiO}_{2}$ interface states in production quality sub-micron devices with excellent sensitivity and resolution. This work should help resolve the ongoing debate as to whether or not $\mathrm{P}_{\mathrm{b}}$ centers are responsible for the "true" electrically measured interface states. Performing the measurements at sufficiently low frequency to ensure complete trap filling separates this method from conventional variable height $\mathrm{CP}$, and is crucial to the accurate extraction of defect density as a function of energy in the band-gap. Furthermore, we have shown that this is a powerful technique for extracting the DOS in other important material systems.

J.T.R. acknowledges funding support by the National Research Council. This work was partially prepared with the 
support of the U.S. Department of Commerce under Award No. NIST 60NANB10D109. However, any opinions, findings, conclusions or other recommendations expressed herein are those of the author(s) and do not necessarily reflect the views of the U.S. Commerce Dept.

\section{REFERENCES}

[1] G. Van den Bosch, G.V. Groeseneken, P. Heremans, and H.E. Maes, "Spectroscopic charge pumping: a new procedure for measureing interface trap distributions on MOS transistors," IEEE Trans. Elec. Dev., vol. 38 (8), pp. 1820-1831 (1991).

[2] J.L. Autran, C. Chabrerie, P. Paillet, O. Flament, J.L. Leray, and J.C. Boudenot, "Radiation-induced interface traps in hardened MOS transistors: an improved charge pumping study," IEEE Trans. Nuc. Sci., vol. 43 (6), pp. 2547-2257 (1996).

[3] J.L. Autran, F. Seigneur, C. Plossu, and B. Balland, "Characterization of $\mathrm{Si}-\mathrm{SiO}_{2}$ interface states: comparison between different charge pumping and capacitance techniques," J. Appl. Phys., vol. 74 (6), pp. 3932-3935 (1993).

[4] M.J. Uren, J.H. Stathis, and E. Cartier, "Conductance measurements on $\mathrm{P}_{\mathrm{b}}$ centers at the (111) $\quad \mathrm{Si} / \mathrm{SiO}_{2}$ interface," J. Appl. Phys., vol. 80 (7), pp. 3915-3922 (1996).

[5] E.H. Poindexter, G.J. Gerardi, M.E. Rueckel, P.J. Caplan, N.M. Johnson, and D.K. Biegelsen, "Electronic traps and $\mathrm{P}_{\mathrm{b}}$ centers at the $\mathrm{Si} / \mathrm{SiO}_{2}$ interface: band-gap energy distribution," J. Appl. Phys., vol. 56 (10), pp. 2844-2849 (1984).

[6] N.M. Johnson, D.K. Biegelsen, M.D. Moyer, S.T. Chang, E.H Poindexter, and P.J. Caplan, "Characteristic electronic defects at the $\mathrm{Si} / \mathrm{SiO}_{2}$ interface," Appl. Phys. Lett., vol. 43 (6), pp. 563-565 (1983).

[7] P.B. Parchinskii, "Density of states at a gamma irradiated $\mathrm{Si} / \mathrm{SiO}_{2}$ interface: the effect of ultrasonic treatment," Solid State Dev. Cir., vol. 34 (6), pp. 420-423 (2005).

[8] Y. Nishioka, E.F. da Silva, and T.P. Ma, "Evidence for (100) $\mathrm{Si} / \mathrm{SiO}_{2}$ interfacial defect transformation after ionizing radiation," IEEE Trans. Nuc. Sci., vol. 35 (6), pp. 1227-1233 (1988).

[9] Y.G. Fedorenko, L. Truong, V.V. Afanas'ev, and A. Stesmans, "Interface state energy distribution in (100) $\mathrm{Si} / \mathrm{HfO}_{2}$," Mat. Sci. Semi. Process., vol. 7, pp. 185-189 (2004).

[10] L.A. Ragnarsson and P. Lundgren, "Electrical characterization of $\mathrm{P}_{b}$ centers in (100) $\mathrm{Si} / \mathrm{SiO}_{2}$ structures: the influence of surface potential on passivation during post metallization anneal," J. Appl. Phys., vol. 88 (2), pp. 938-942 (2000).

[11] J.L. Autran, C. Plossu, F. Seigneur, B. Balland, "A comparison of $\mathrm{Si} / \mathrm{SiO}_{2}$ interface trap properties in thin film transistors with thermal and plasma nitrided oxides," J. Non-Crys. Solids, vol. 187, pp. 374-379 (1995).

[12] Y. Wang, T.P. Ma, and R.C. Barker, "Orientation dependence of interface trap transformation," IEEE Trans. Nuc. Sci., vol. 36 (6), pp. 1784-1791 (1989).
[13] P.K. Hurley, A. Stesmans, V.V. Afanasev, B.J. O'Sullivan, and E. O'Callaghan, "Analysis of $\mathrm{P}_{\mathrm{b}}$ centers at the $\mathrm{Si}(111) / \mathrm{SiO}_{2}$ interface following rapid thermal annealing," J. Appl. Phys., vol. 93 (7), pp. 3971-3973 (2003).

[14] N. Haneji, L. Vishnubhotla, and T.P. Ma, "Possible observation of $\mathrm{P}_{\mathrm{b} 0}$ and $\mathrm{P}_{\mathrm{b} 1}$ centers at irradiated (100) $\mathrm{Si} / \mathrm{SiO}_{2}$ interface from electrical measurements," Appl. Phys. Lett., vol. 59 (26), pp. 3416-3418 (1991).

[15] P.M. Lenahan and J.F. Conley, "What can electron paramagnetic resonance tell us about the $\mathrm{Si} / \mathrm{SiO}_{2}$ system," J. Vac. Sci. Technol. B, vol. 16 (4), pp. 2134-2153 (1998).

[16] E. Cartier and J.H. Stathis, "Atomic hydrogen induced degradation of the $\mathrm{Si} / \mathrm{SiO}_{2}$ structure," Microelectron. Eng., vol. 28, pp. 3-10 (1995).

[17] G. Groeseneken, H.E. Maes, N. Beltran, and R.F. De Keersmaecker, "A reliable approach to charge pumping measurements in MOS transistors," IEEE Trans. Elec. Dev., vol. 31 (1), pp. $42-53$ (1984).

[18] http://www-device.eecs.berkeley.edu/qmov/index.shtml, This document includes links to websites that may have information of interest to the reader. NIST does not necessarily endorse the views expressed or the facts presented on these sites. Further, NIST does not endorse any commercial products that may be advertised or available on these sites.

[19] A. Savitzky and M.J.E. Golay, "Smoothing and differentiation of data by simlified least squares procedures," Anal. Chem., vol. 36 (8), pp. 1627-1639 (1964). 